\title{
Paint Effects on the Advanced Quantitative Infrared Thermography Applied to Jet Impacts
}

by R. Mehryar, A. Giovannini and S. Cazin

Institut de Mécanique des Fluides, Toulouse, France

\begin{abstract}
The effect of a paint layer on a heated thin stainless steel foil for heat transfer coefficient calculation is studied. The foil is cooled by a jet impact ( $R e=100$ to 1000$)$ on one side and it is observed by an infrared camera on the other painted side. The results show that the lateral conduction has an important role in heat transfer calculation and should be considered the thickness and the thermal properties of the paint in the energy balance equations. In addition, the paint quality and the camerafoil distance have an important role in the spatial noise level for calculated conduction term calculation.
\end{abstract}

\section{Introduction}

Over the last 20 years infrared thermography has evolved into a powerful investigative means of thermo-fluid-dynamic analysis to measure convective heat flux. Most of potential error sources, linked to the object, the environment and the acquisition system, which may affect temperature measurement have been understood, eliminated or taken into account [1]. A current application of infrared thermography is temperature measurement of a heated thin foil that is cooled by jet impact. The opposite side of the foil, in front of the camera, is covered by a high emmisivity paint layer, but the authors did not discuss precisely its effect on the heat transfer calculation. Some of them calculated the temperature on the impact side of the impingement plate from the infrared temperature measurement of the opposite side [2]. The others assumed that the temperature on the two sides to be the same as the Biot number is very small $(<0.1)[3,4]$ or they estimated their difference as negligible compared to the temperature difference between the plate and the jet flow [5].

In our research an experimental apparatus was designed in order to study the flow and heat transfer over multiple air jets impacting in a confined area. But we encountered several problems for calculation of heat transfer coefficient relating to the paint layer. So in this paper we try to investigate the effect of paint and its thickness on the heat transfer calculation. So at first we will show the calculation method of the heat transfer coefficient by the temperature field measured with an infrared camera and also the difficulty in the calculation of the lateral heat conduction term. Then we will check this calculation method by a numerical code, prepared by the authors. Finally we will present the modified equations used for evaluating the convection heat transfer coefficient by considering the paint layer.

\section{Measurement method}

The experimental apparatus was composed of two plates, impingement and jet plates. The jet plate was made of Aluminium with 9 holes of $3 \mathrm{~mm}$ in diameter in a square in line array. It was also possible to install a plate with only one hole instead 
of it. The impingement plate was made of nylon and in its central part a thin stainless steel foil was embedded (figure 1) that was heated by joule effect. The foil was cooled by the air jet flow in the lower side and it was exposed to the ambient air by the higher side. The measurements were made in steady state and also in transient conditions. In the later the jet flow existed but the foil was heated abruptly by a computer controlled electric source. So there are the radiation heat losses from both sides and the natural convection heat losses from the higher side. The thermal cartography was obtained by infrared thermography with a camera JADE III MWIR working in the range of $3 \mu \mathrm{m}<\lambda<5 \mu \mathrm{m}$. It measured the radiation emitted by the foil in "digital level" unit. For calibration of the camera, a uniform and precise temperature source was needed. A black body source of DCN 100 was used. The data acquisition and first post processing were made by ALTAIR software for steady state and also transient conditions. The schematic of the infrared camera and the black body utilization is shown in figure 2. The camera saw the back side of the foil that is covered by several layers of a black paint whose emissivity had been characterized at the value of 0.94 [6].

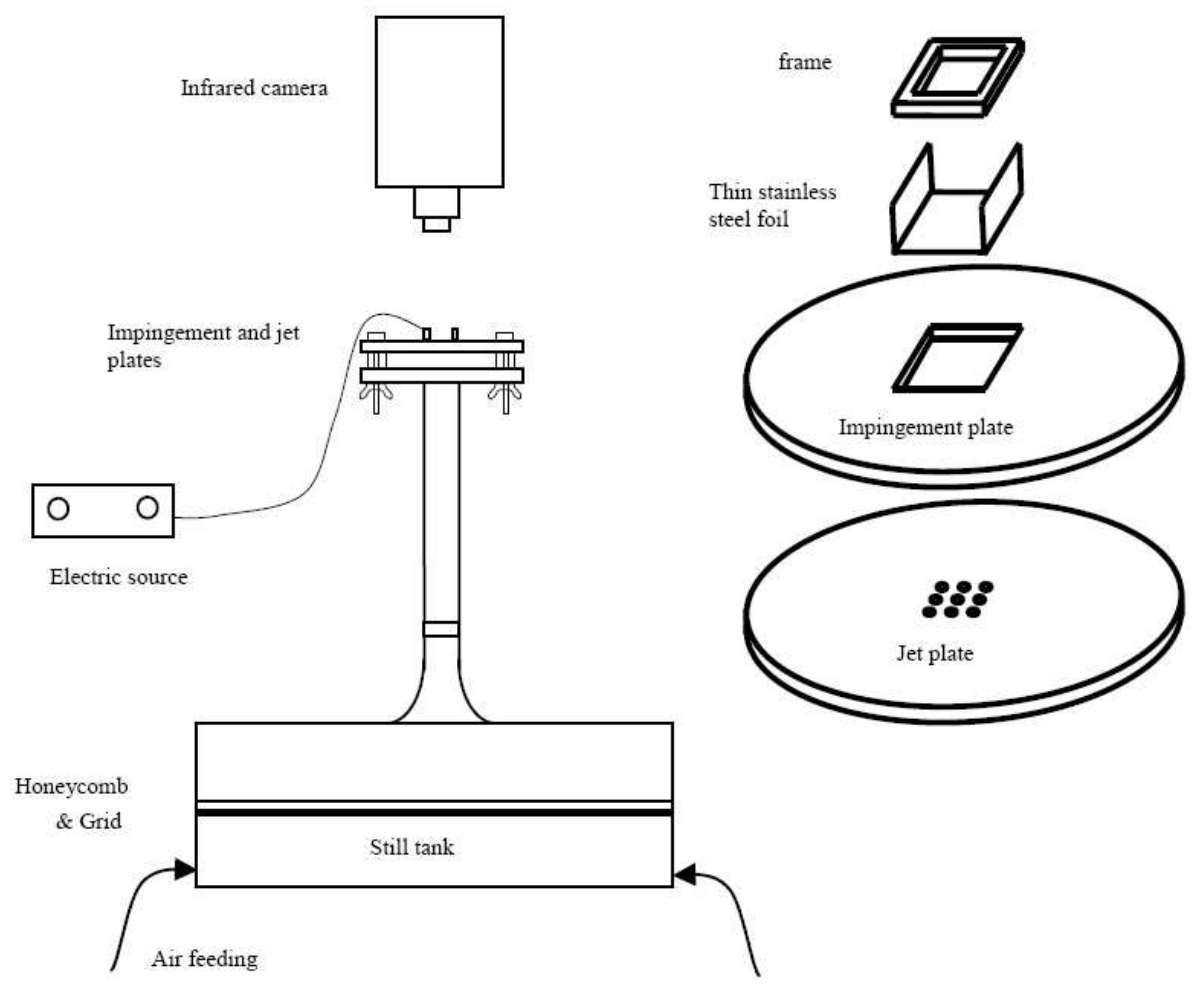

Fig. 1. Simplified schematic of the experimental apparatus 


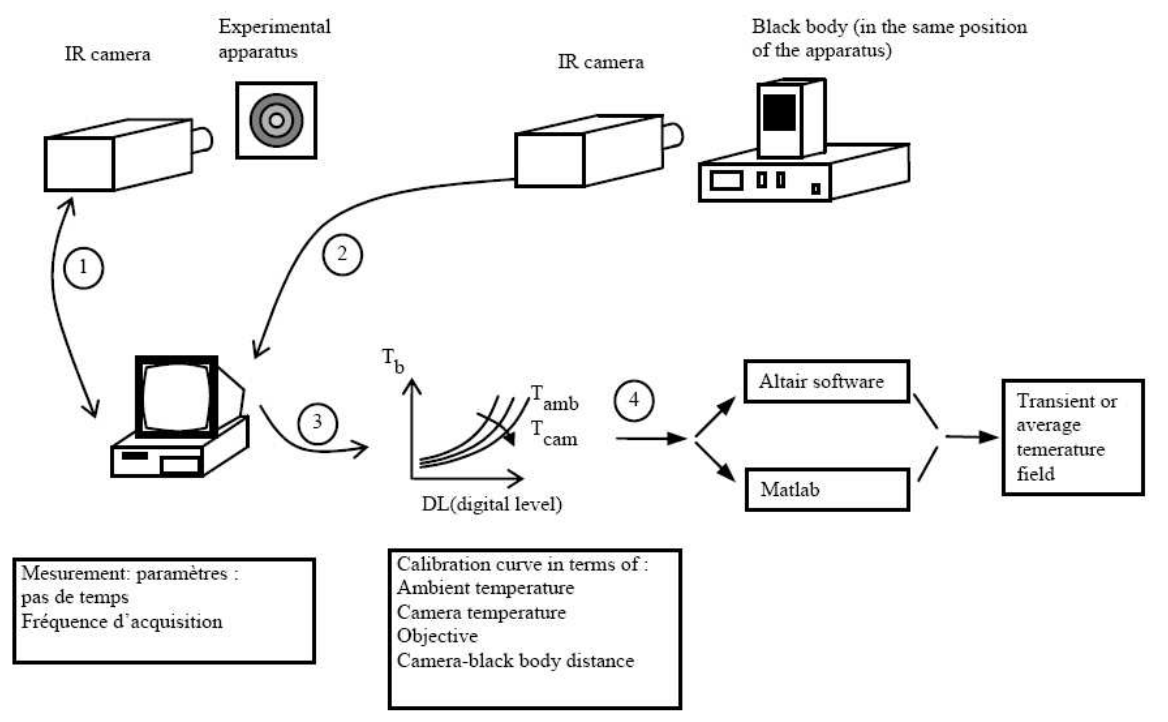

Fig. 2. Infrared measurement procedure

\section{Calculation method}

In order to obtain heat transfer coefficient from the measured temperature field, the energy balance equation (1) is used for each element of the foil.

$Q_{\text {jet }}=Q_{\text {gen }}-Q_{\text {st }}-Q_{\text {cond }}-Q_{\text {loss }}$

Each element has the foil thickness, e, and the surface of a pixel ( $A_{p i x}=\Delta x \Delta y$ ).

The different terms of this equation are computed by the Eqs. (2) to (5). The heat generation term, $Q_{\text {gen }}$ is obtained by Eq. (2).

$Q_{\text {gen }}=\frac{E I}{L l} A_{p i x}$

where $E$ and $I$ are the voltage and the current passing the foil of width and length of $l$ and $L$. For the transient conditions, the storage term is obtained by:

$Q_{s t}=\rho c e \frac{T_{i, j}^{n+1}-T_{i, j}^{n-1}}{\Delta t} A_{p i x}$

The conduction term can be calculated at each pixel, situated at the position (i,j), by using a pixel at a distance of $m \Delta x$ and $m \Delta y$. 
$Q_{\text {cond }}=-k e\left(\frac{T_{i+m, j}^{n}-2 T_{i, j}^{n}+T_{i-m, j}^{n}}{m^{2}(\Delta x)^{2}}+\frac{T_{i, j+m}^{n}-2 T_{i, j}^{n}+T_{i, j_{-} m}^{n}}{m^{2}(\Delta y)^{2}}\right) A_{p i x}$

Heat losses from the foil are in the form of radiation and natural convection, and in this situation it can be presented totally by Eq. (5)

$$
Q_{\text {loss }}=h_{\text {loss }}\left(T_{i, j}^{n}-T_{a m b}\right) A_{p i x}
$$

So by calculating all of the right hand terms in Eq. (1), the heat transfer coefficient is obtained by:

$$
h_{j e t}=Q_{j e t} /\left(T_{i, j}^{n}-T_{j e t}\right) / A_{p i x}
$$

The heat transfer coefficient, obtained by these equations, seems accurate due to the low values of Biot number for stainless steel foil and paint layer. Nevertheless we encountered the problems in the heat transfer calculation, so we will evaluate different parameters by a numerical inverse method that will be explained and discussed in the next part. It is noted that by replacing different terms of Eqs. (2) to (4) in Eq. (1) we can present this equation in the differential form.

$$
\rho c \frac{\partial T}{\partial t}=k\left(\frac{\partial^{2} T}{\partial x^{2}}+\frac{\partial^{2} T}{\partial y^{2}}\right)-\frac{q_{j e t}+q_{\text {loss }}}{e}+\frac{E I}{L l e}
$$

In these equations the unit of ' $q$ ' is $W / m 2$, while the unit of ' $Q$ ' is $W$.

\section{$4 \quad$ Simulating program}

For finding different effects of the paint layer on the calculation of heat transfer coefficient we simulated the equation of three dimensional transient conduction heat transfer (Eq. (8)).

$$
\rho c \frac{\partial T}{\partial t}=k\left(\frac{\partial^{2} T}{\partial x^{2}}+\frac{\partial^{2} T}{\partial y^{2}}+\frac{\partial^{2} T}{\partial z^{2}}\right)+g
$$

This equation is used for the foil and also for the paint layer with its thermophysical characteristics and the heat generation value equals to zero. In order to discretize this equation, we used the technique ADI (Alternating direction method), developed by Douglas-Gunn (for more information see $[7,8]$ ). This Cranck-Nicolson method is unconditionally stable and has a second order accuracy in time and space. So it simplifies the calculation procedure.

A simulated forced convection boundary condition, (Eq. (9)), is considered on the jet impact side of the foil.

$$
h_{j e t}=h_{\text {min }}+\left(h_{\text {max }}-h_{\text {min }}\right) \exp \left(-a_{0}^{2}\left(x^{2}+y^{2}\right) / D^{2}\right)
$$


This formula produces a heat transfer coefficient profile that is similar to that of one jet and varies from $h_{\max }=200 \mathrm{~W} / \mathrm{m}^{2} \mathrm{~K}$ at the centre of foil corresponding to jet centr $\mathrm{e}$ to $h_{\min }=10 \mathrm{~W} / \mathrm{m}^{2} \mathrm{~K}$ for $\mathrm{x} / \mathrm{D}$ higher than 6 . The boundary condition in the opposite side of the foil is considered as the natural convection with $h=5 \mathrm{~W} / \mathrm{m}^{2} \mathrm{~K}$. The contact conditions between the foil and the paint layer was considered perfect with continuity of the temperature and the heat flux, as in Eq. (10).

$$
\left.\left.T_{p a \text { int }}=T_{\text {foil }} \quad \text { and }-k \frac{\partial T}{\partial z}\right)_{p a \text { int }}=-k \frac{\partial T}{\partial z}\right)_{\text {foil }}
$$

where $\mathrm{k}$ is thermal conductivity. It is equal to $16.3 \mathrm{~W} / \mathrm{mK}$ for the foil and $1 \mathrm{~W} / \mathrm{mK}$ for the paint [9].

Using these equations, we can obtain the temperature field. So it is possible to investigate the effect of thickness and thermo-physical properties of the paint on the temperature field.

In addition, with the temperature field, obtained from the ADI code, we used the Eq. (1) to (5) to calculate the jet heat transfer coefficient by Eq. (6). The results should be corresponding to the Eq (9). By this method we could find the errors existing in the formulas (2) to (5), the equations that every one used with the infrared thermography.

\section{$5 \quad$ Results}

\subsection{Infrared measurement}

The temperature field for nine jets in square in line array is shown in figure 3 . The reason of the dissymmetry shape is not the objective of this paper and it was discussed in another paper [10]. This figure was obtained in the steady state conditions by averaging the temperature field of about hundred images. Thus all of the temporal noises were attenuated. Nevertheless if we calculate the conduction term we will observe a considerable noise that is very higher than the correct conduction profile (figure 4). This figure is corresponding to the temperature profile, shown in figure 5. For solving this problem, an experimental and numerical modification should be applied. For the experimental side, it should be concentrated on the paint quality, the camera-to-plate distance and the heating level of the foil. The paint quality has an important role in the spatial noise level of the temperature field, measured by the camera. If the paint grains on the foil are distinguished by the camera or if the foil is contaminated or degraded, the noise increases rapidly. The foil can be contaminated by the suspended particles in air produced by the PIV measurements [11], or can be degraded by the external objects in contact with the foil. Another factor that can decrease the noise level is the camera-to-plate distance (figure 6). Increasing this distance without changing the lens of camera, the camera sees less the paint grain on the foil, but in the same time the number of points in the jet diameter is decreased which limits the spatial resolution. Increasing the temperature of the heated foil decreases generally the uncertainty of the results, but when the observed surface is not completely smooth the results will be inversed (figure 7). So for the two last parameters, it should be selected the optimum values with a compromise between the explained criteria.

For the numerical side, we can filter the temperature field and obtain the temperature for each pixel by averaging it with the $3^{*} 3$ or $5^{\star} 5$ pixels. Then for calculating the conduction term by Eq. (4) we consider $m$ equal to 3 or 5 or even 7 if the temperature gradient is not high. Therefore the results of these modifications give a 
conduction term which is shown in figure 8 and compared to the other terms of Eq. (1).

After the filtering and obtaining the acceptable results for the profile of heat transfer coefficient, we tried to calculate the heat transfer coefficient in transient conditions by the Eqs. (1) to (6). But the results were not logical. The values obtained in transient conditions were severely greater than the steady state results. So we analyse the calculation method numerically in the next section.

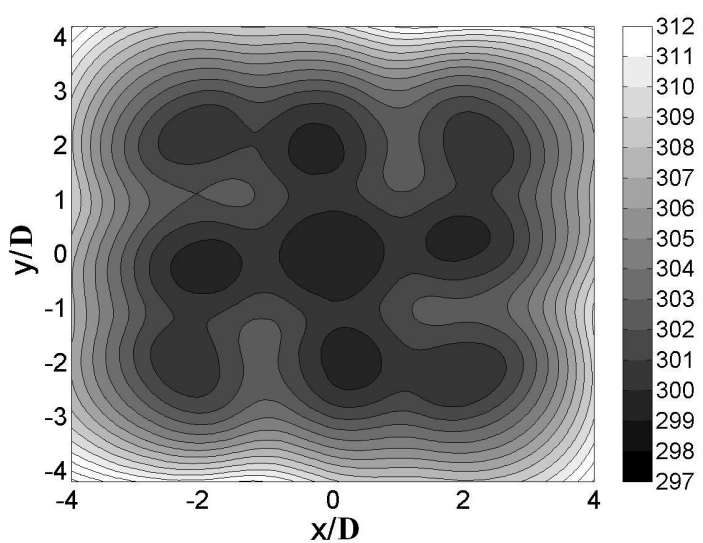

Fig. 3. Temperature field for 9 jets in square in line array

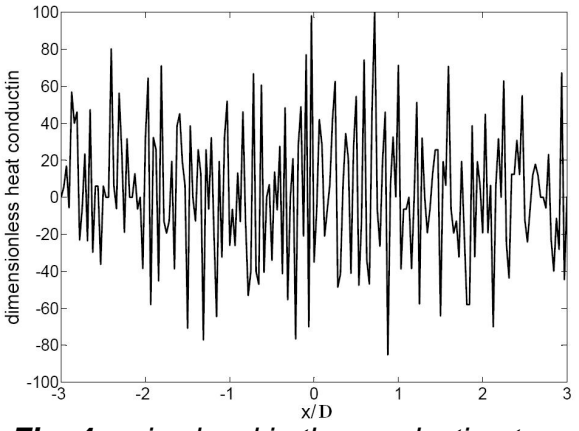

Fig. 4. noise level in the conduction term

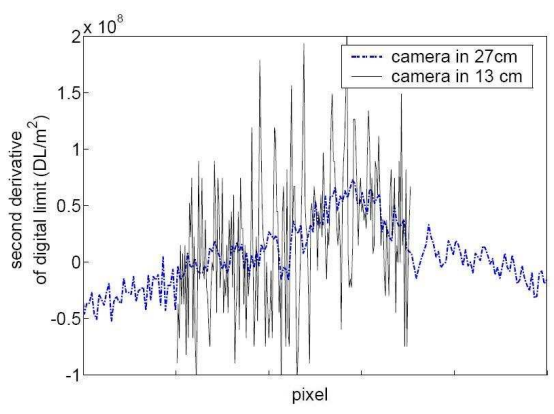

Fig. 6. Effect of camera-to-plate distance on the noise level

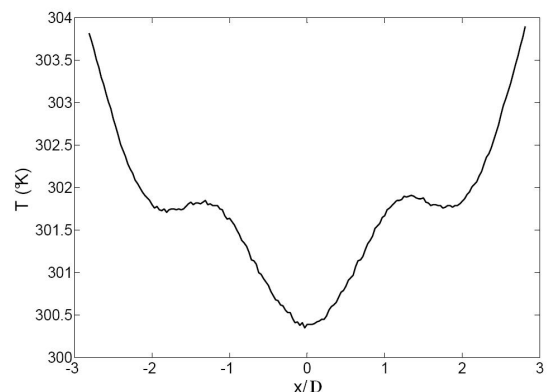

Fig. 5. temperature profile on the $x$ axis

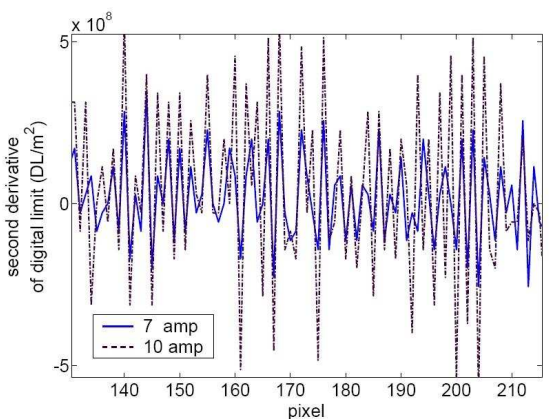

Fig. 7. Effect of foil heating level on the noise level 


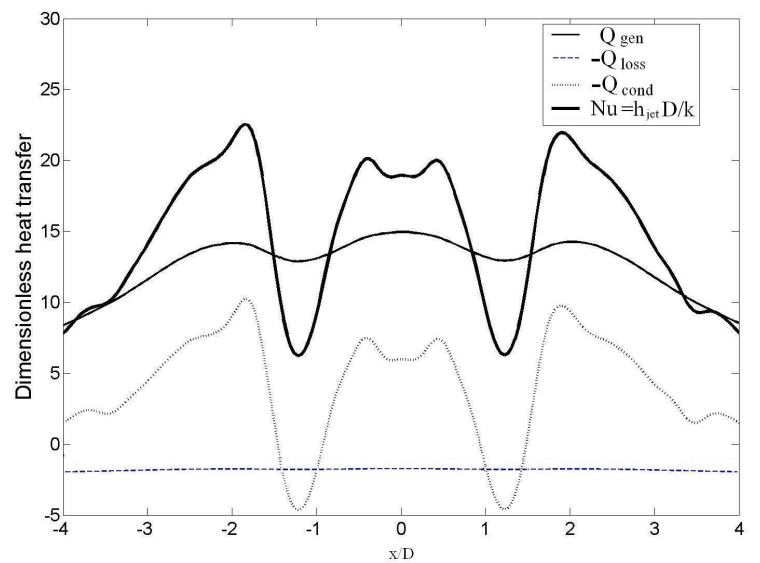

Fig. 8. Different heat transfer terms obtained after experimental and numerical modifications

\subsection{Simulation}

For evaluating the heat transfer calculation method, presented in Eqs. (1) to (6), we used the ADI method with the boundary condition of forced convection heat transfer Eq. (9). In the first step, with a direct calculation method and by considering different thicknesses and various thermal conductivities for the paint, the corresponding temperature field are obtained and compared in figures 9 and 10. Figure 9 shows that if the paint thermal conductivity is in the same order of magnitude of the foil thermal conductivity $(k=16 \mathrm{~W} / \mathrm{mK})$, the temperature field will be changed considerably. This variation is happened because of the lateral conduction in the paint layer. On the other side, increasing the paint thickness has not large influence on the temperature field. The maximum temperature difference between the cases of $40 \mu \mathrm{m}$ and $200 \mu \mathrm{m}$ thickness is equal to $0.2 \mathrm{~K}$ in the jet centre. So until this part of the results, we conclude that the small variations of the paint thickness and its thermal conductivity make no large differences on the temperature field.

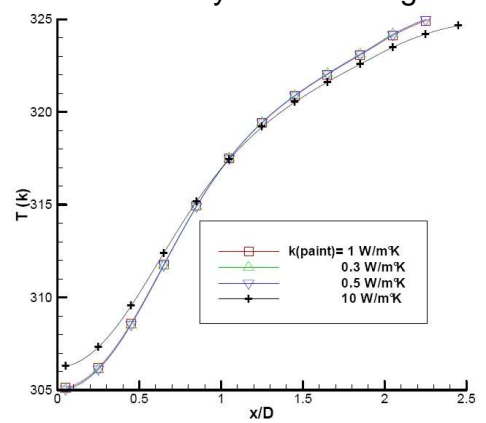

Fig. 9. Effect of paint thermal conductivity on the temperature field

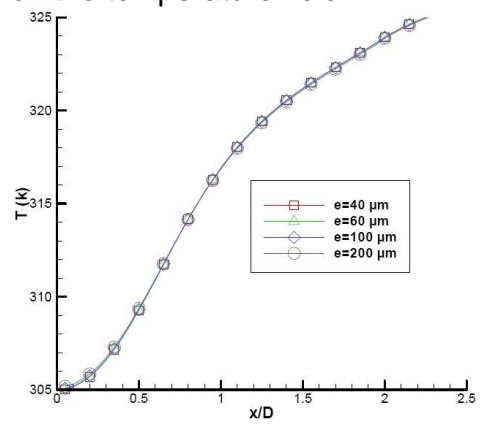

Fig. 10. Effect of paint thickness on the temperature field

The second step of evaluation is the recalculation of boundary condition (Eq. (9)), from the temperature field obtained in the first step and by using Eqs. (1) to (6). For this means we choose two different points (P1 \& P2) on the plate at $x / D$ equal to 0.17 and 4.2. The corresponding global heat transfer coefficients at P1 and P2 are 203.1 
and $15.5 \mathrm{~W} / \mathrm{m}^{2} \mathrm{~K}$ respectively, with taking into account the natur al heat transfer coefficient that was considered at the opposite side of the foil. By the inverse method the value of heat transfer coefficient obtained in the short time is very higher than the steady state value. We obtained the same trends with processing the experimental data of infrared thermography. For finding the reason of this difference, these two steps were used for several paint thicknesses and different numbers of nodes in it. The results are shown in figure 11. As it is seen, changing the number of nodes in the direction of the thickness of the foil and paint (different $\Delta z$ ) has no effect on the results. So the numerical grid size was chosen properly and the obtained value for heat transfer coefficient has no numerical error. But in addition to the deviations at the short time, it is observed a difference between the steady state values for different thicknesses.
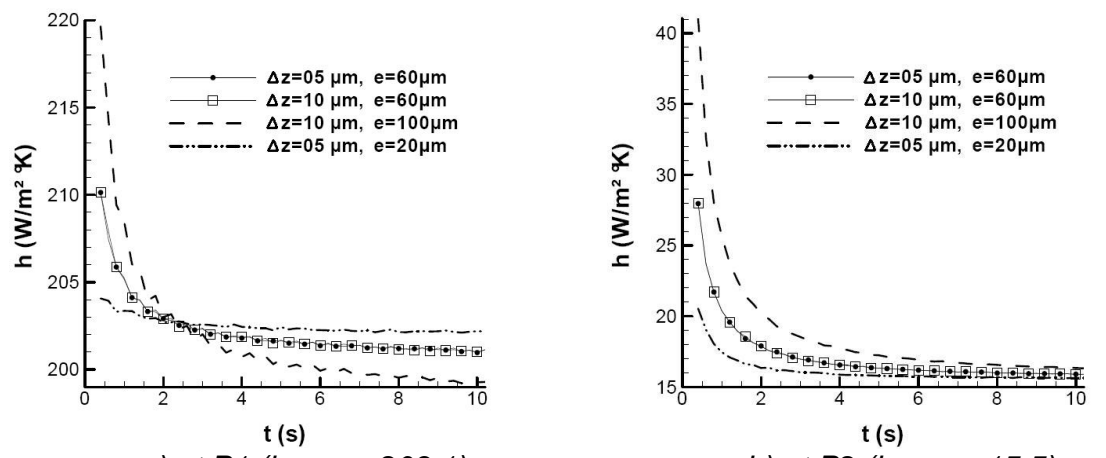

a) at $P 1\left(h_{\text {exact }}=203.1\right)$

b) at $P 2\left(h_{\text {exact }}=15.5\right)$

Fig. 11. Heat transfer coefficient obtained by indirect method

Another simulation test was made for being sure that the observed deviations were due to the paint layer. In this simulation the paint layer is eliminated and the same procedure is carried out for obtaining heat transfer coefficient. The results are compared to the last case (figure 12). It is clear that the deviation is due to the paint layer and so it should be considered the conduction and also storage terms in it.

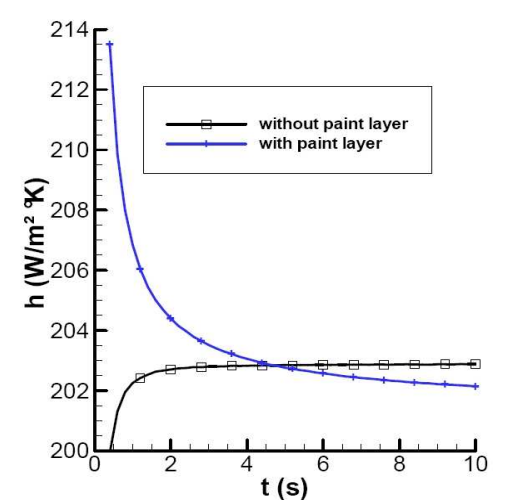

a) at $P 1$

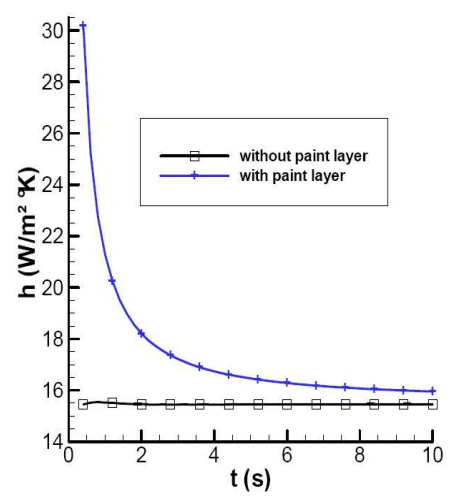

b) at $P 2$

Fig. 12. Comparison of the obtained heat transfer coefficient with and without paint layer 
For solving the problem we use the energy equation, Eq. (7), for each layer with the related thermal properties.

$$
\begin{aligned}
& \rho_{f} c_{f} \frac{\partial T}{\partial t}=k_{f}\left(\frac{\partial^{2} T}{\partial x^{2}}+\frac{\partial^{2} T}{\partial y^{2}}\right)-\frac{q_{\text {jet }}+q_{\text {contact }}}{e_{f}}+\frac{E I}{\text { Lle }_{f}} \\
& \rho_{p} c_{p} \frac{\partial T}{\partial t}=k_{p}\left(\frac{\partial^{2} T}{\partial x^{2}}+\frac{\partial^{2} T}{\partial y^{2}}\right)-\frac{q_{\text {loss }}-q_{\text {contact }}}{e_{p}}
\end{aligned}
$$

If the Biot number corresponding to the paint layer and the heat loss coefficient of the paint side is low $(<0.01)$, it will be possible to consider the same temperature field for the foil and paint layer at each time. So we can eliminate the $q_{\text {contact }}$ between these two equations and the Eq. (13) is obtained.

$$
\left(\rho_{f} c_{f}+\frac{\rho_{p} c_{p} e_{p}}{e_{f}}\right) \frac{\partial T}{\partial t}=\left(k_{f}+\frac{k_{p} e_{p}}{e_{f}}\right)\left(\frac{\partial^{2} T}{\partial x^{2}}+\frac{\partial^{2} T}{\partial y^{2}}\right)-\frac{q_{j e t}+q_{\text {loss }}}{e_{f}}+\frac{E I}{L l e_{f}}(13)
$$

The assumption of temperature equality is valid for the case of this research where the Biot number for the paint layer is about 0.001 .

So by correcting the coefficients of Eqs. (2) \& (3) according to Eq. (13), we obtained the same results for heat transfer coefficient in the steady state and transient conditions and also for different thickness (figure 13). It is concluded that for calculating heat transfer coefficient, the thermal conductivity and volumetric heat capacity of the foil should be modified as in Eq. (13). The corresponding modifications of these two parameters are $7 \%$ and $4 \%$ respectively.

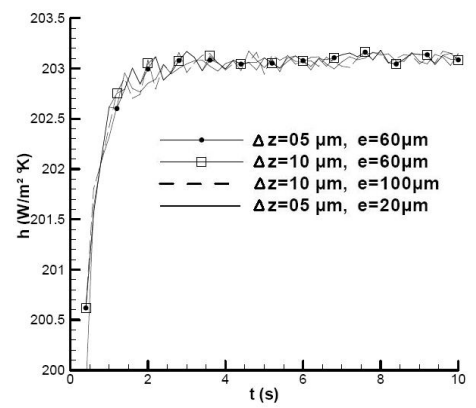

a) at $P 1$

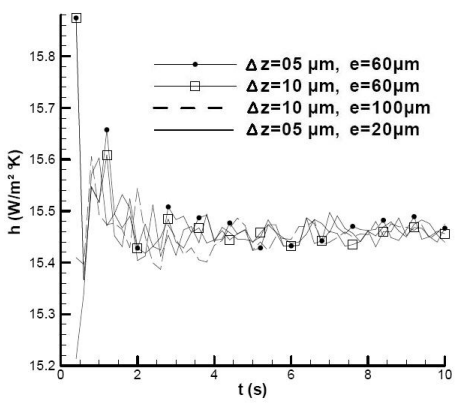

b) at $P 2$

Fig. 13. Heat transfer coefficient obtained after the correction 


\section{Conclusion}

The paint quality and the camera-to-foil distance have an important role in the spatial noise level of the temperature field, measured by the camera. If the paint grains on the foil are distinguished by the camera or if the foil is contaminated, and/or if the camera is too near to the impingement plate, the noise level will increase. On the other hand, by increasing the distance, the noise decreases but there will not be enough points in the jet diameter to evaluate the profile of heat transfer coefficient. Therefore a compromise forced us to place the camera at a distance optimum. In addition, the equality of the temperature field on the impacted side and the paint side is not sufficient for neglecting the paint effect in the heat transfer calculation. Although the temperature field on the foil does not change too much for different paint thicknesses or various thermo-physical properties of the paint, but the lateral heat conduction and transient terms in the paint layer are not negligible in comparison with the equivalent terms in the foil layer. So the thermal conductivity and the volumetric heat capacity of the foil should be modified with the same terms of the paint layer multiplied by the thickness ratio of the paint and the foil.

\section{REFERENCES}

[1] A. Astarita, G. Cardone, G.M. Carlomagno and C. Meola. A survey on infrared thermography for convective heat transfer measurements, Optic \& Laser Technology, 32 (2000) 593-610.

[2] P. Brevet, C. Dejeu, E. Dorignac, M. Jolly and J.J. Vullierme. Heat transfer to a row of impinging jets in consideration of optimization, International Journal of Heat \& Mass Transfer, 45 (2002) 4191-4200.

[3] D. Lytle and B.W. Webb. Air jet impinging heat transfer at low nozzle-plate spacings, International Journal of Heat \& Mass Transfer, 37 (1994) 1687-1697.

[4] D. Varieras and P. Gervais. Détermination du coefficient de transfert convectif entre un jet et une surface: analyse de la qualité de la mesure, VIlème Colloque Interuniversitaire Franco-Quebecois sur le Thermique de Systèmes, (2005) 301-305.

[5] Y. Pan, J. Stevens and B.W. Webb. Effect of nozzle configuration on transport in the stagnation zone of axisymmetric, impinging free surface liquid jets: Part2local heat transfer, ASME Journal of Heat Transfer, 114 (1992) 880-886.

[6] N.S. Kim. Analyse expérimentale d'un jet turbulent impactant sur une plaque plane et sur un obstacle de section carrée, PhD Thesis (2005).

[7] R. Mehryar and A. Giovannini. Interaction entre jets multiples impactant en milieu confiné, VIlème Colloque Interuniversitaire Franco-Quebecois sur le Thermique de Systèmes, (2005) 289-294.

[8] T.Y. Wang and C.C.P. Chen. 3-D thermal ADI: a linear-time chip level transient thermal simulator, IEEE Transaction on Computer-Aided Design of Integrated Circuits and Systems, 21 (2002) 1434-1445.

[9] T.J. Praisner, D.R. Sabatino and C.R. Smith. Simultaneously combined liquid crystal surface heat transfer and PIV flow-field measurements, Experiments in Fluids, 30 (2001) 1-10.

[10] R. Mehryar and A. Giovannini. Thermal evaluation of jet-to-plate spacing effects in laminar confined multijets, 13th International Heat Transfer Conference, Australia, (2006) In press.

[11] M. Raffel, C.E. Willert and J. Kompenhass. Particle image velocimetry - A practical guide, Springer (1998). 